\title{
Flying Posture of Tandem Warhead Influences its Penetrating and Following Performance
}

\author{
Jin-tao Wang*, Wen-li Yu and Tao Wang \\ Dept. of Engineering Physics, Xi'an Research Inst. Of Hi-Tech, Xi'an, Shaanxi 710025 - China
}

Received 20 October 2013; Accepted 25 February 2014

\begin{abstract}
In this study, we investigated a novel monolithic tandem penetrating-blast warhead, which can destroy targets with adjustable parameters including attack velocity, angles, as well as yaw angles. Different flying postures determine different performance of the precursory EFP in penetration process and its following projectile in secondary process. In order to characterize the influence of flying posture, we established a finite elements analysis model. This model has been verified by a static experiment. Our results indicated that the attack velocity of the warhead has tiny influence of the penetration process. However, larger attack angle of the warhead will lead to bad performance of the warhead. Meanwhile, yaw angle range should be controlled in small values due to its randomness. The comprehensive properties of the tandem warhead meet the design requirement, and it is able to damage the target effectively.
\end{abstract}

Keywords: Mechanics of explosion; Tandem warhead; Flight attitude; Penetration; Numerical simulation

\section{Introduction}

In the past few years, the tandem warhead has attracted lot of attention and underwent rapidly development. The structure form appears diversified gradually. Especially, the continuous application of new technology and new materials makes tandem warhead be a powerful weapon against complex solid targets in a modern campaign. Shaped cut blasting tandem warhead contains precursor shaped device, secondary blasting destructive projectile, and mainly targets large naval vessels with multi-layer septal deck structure. Attacking way as follows: the explosively formed projectile (EFP) produced by shaped effect of precursor device penetrates the top deck and subsequently produces a hole; the secondary projectile follows across the hole and penetrates the subsequent decks rely on its own kinetic energy; then, according to the preset program, the secondary projectile timely explodes, to destroy the valuable targets effectively and massively inside the vessels [1-9].

Because of its complicated structure, the structure design, function sequence, and damage efficiency between precursor device and secondary projectile must achieve efficient matching, in order to show the power of the tandem warhead and achieve damage intentions.

The structure was determined based on optimization design and damage performance investigation of both precursor device and secondary projectile. Then, the structure of the whole warheads was determined. In order to match the two components reasonably and scientifically, the distance between precursor device and secondary projectile was determined, and after that, we studied the penetration and following performance.

- E-mail address: wangiintaolove@126.com

ISSN: 1791-2377 @ 2014 Kavala Institute of Technology. All rights reserved.
In this paper, we focused on the matching performance of tandem warhead between two processes: precursor EFP penetrating and secondary projectile following across the top deck. Attack velocity, angle and yaw angle are the three major influence factors involved in the two processes. Using the finite element code, LS-DYNA, we established a simulation model and verified it by static scaled-down experiments, in order to obtain reliable model parameters. We characterized the influence rules of the three factors using a model developed by LS-DYNA.

\section{Computational details}

\subsection{Finite elements model}

Finite elements (FE) method is a numerical technique for finding approximate solutions to real complicated problems. LS-DYNA is a general-purpose finite element program capable of simulating complex real world problems. It is used by the automobile, aerospace, construction, military, manufacturing, and bioengineering industries. LS-DYNA is optimized for shared and distributed memory Unix, Linux, and Windows based, platforms. The code's origins lie in highly nonlinear, transient dynamic finite element analysis using explicit time integration.

The numerical simulation of a tandem warhead (see fig.1) requires the description of the penetration process of the shaped charge jet and the KE projectile as well as their interaction. The most important phenomena are:

Shaped charge jet formation (EFP);

Erosion of EFP and target during penetration;

Crater formation including damage of metal target;

Secondary projectile penetration (nearly rigid body penetration). 
In order to improve the calculation efficiency, the FE model of the precursor shaped device was simplified into three components: liner, high-energy explosive (HE) and case. The fuze was transformed to set a detonation point in the model, as shown in figure 1 . The secondary projectile is filled with high-energy explosive inside, while, the fuze and other components were not considered in this model system. The relative position between precursor shaped device and secondary projectile is identical to real situation.

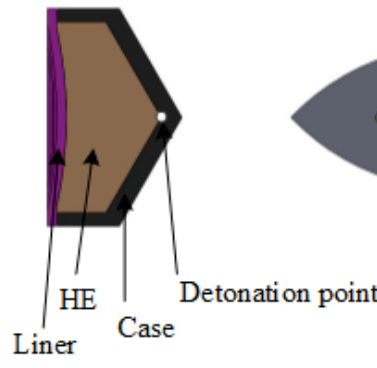

(a) Former Class

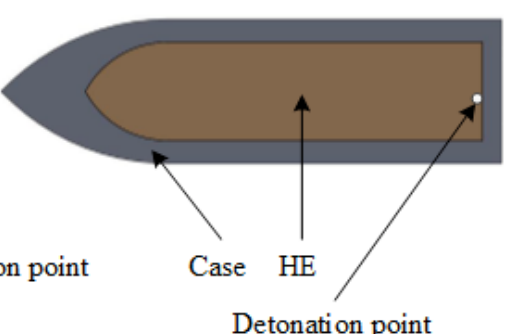

(b) After Class
Fig.1. Geometric model of warhead

The assessment of the tandem system efficiency combines the effects of the precursor shaped charge and the secondary projectile. Determined by the structural characteristics of vessels' structure properties and the attacking position of the tandem warhead, the penetration and secondary processes only involve the top deck of vessels. So, the simulation model of the $60 \mathrm{~mm}$ thick top deck was established. The precursor shaped device was detonated when its front point is $0.9 \mathrm{~m}$ away from the top deck.

The elements meshing of the warhead and top deck show in figure 2 and 3. Because of the simulation of the precursor shaped device involves an explosive and huge deformation; therefore, fluid-structure interaction method was used. The explosive model was meshed with Eulerian algorithm, and the liner and case models were meshed with Lagrangian algorithm. Two types of elements were coupled together during the simulation calculation, as shown in figure 2(a). The whole secondary projectile was meshed with Lagrangian algorithm, as shown in figure 2(b). The top deck was also meshed with Lagrangian algorithm, and its diameter is 5 times of the warhead (Actually, the top deck is much larger). Considering the impact area is much smaller than the top deck, the size of elements transferred from small ones in the center to large ones around, as shown in figure 3. Non-reflection condition was applied to the deck boundary. Due to it has the plane of symmetry, we only established half of the simulation model.

Fluid-structure interaction (FSI) is the interaction of some movable or deformable structure with an internal or surrounding fluid flow. Arbitrary Lagrangian Eulerian (ALE) finite element methods gain interest for the capability to control mesh geometry independently from material geometry. In fluid-structure interaction problems, where the fluid mesh near the structure undergoes large deformations and becomes unacceptably distorted, which drive the time step to a very small value for explicit calculations, the ALE methods or rezoning are used to create a new undistorted mesh for the fluid domain, which allows the calculations to continue. The mathematical basis of the ALE and rezoning algorithms is simple, but their implementation is complicated due to the tedious geometrical calculations associated with handling an arbitrary mesh.
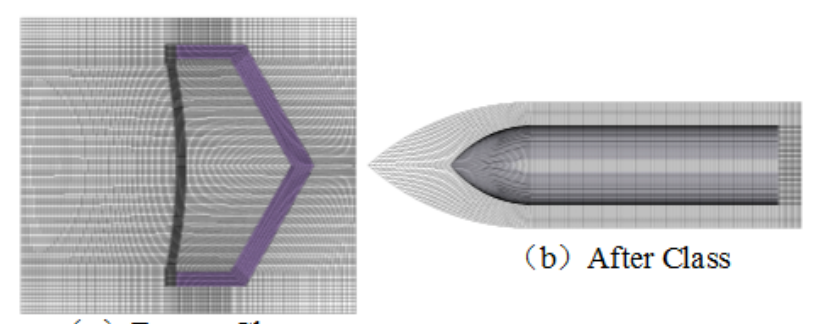

(b) After Class

(a) Former Class

Fig.2. Elements meshing of the warhead

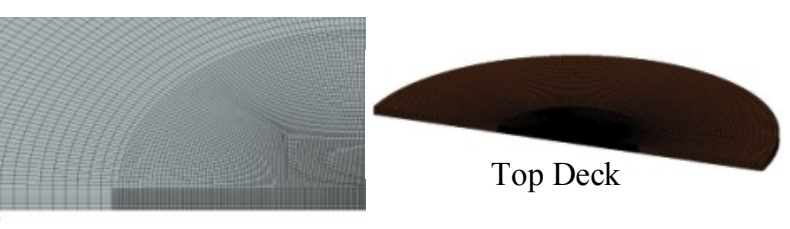

Partial Enlargement

( Top Deck)

Fig.3. Elements meshing of the top deck

\subsection{Materials model}

The simulation contains the six components: liner, case, charge of precursor shaped device, steel body and inner charge of secondary projectile, and top deck of vessels. The liner, case, and charge of precursor shaped device are described with Steinberg model, Johnson Cook model, and High Explosive Burn model, respectively. Very important is the material description of the secondary projectile body and top deck, especially the weakening of the target due to the passage of the shaped charge jet. Therefore, They are described with Plastic Kinematic model.

Materials models used in this paper are shown in table 1, and the values of their parameters were obtained from experiments.

Table 1. Material models of each component

\begin{tabular}{|c|c|c|}
\hline Component Name & Material & Material Model \\
\hline Precursor Liner & Copper & $\begin{array}{l}\text { STEINBERG } \\
\text { (GRUNEISEN) }\end{array}$ \\
\hline Precursor Case & Steel & $\begin{array}{l}\text { JOHNSON_COOK } \\
\text { (GRUNEISEN) }\end{array}$ \\
\hline Precursor Charge & $\mathrm{HE}$ & $\begin{array}{l}\text { HIGH_EXPLOSIVE_BURN } \\
\text { (JWL) }\end{array}$ \\
\hline $\begin{array}{l}\text { Secondary } \\
\text { projectile }\end{array}$ & 30CrMnSi Steel & PLASTIC_KINEMATIC \\
\hline Top Deck & Steel & PLASTIC_KINEMATIC \\
\hline
\end{tabular}

For the Steinberg model, we define the shear modulus, $G$, before the material melts as:

$$
G=G\left[1+b p V^{\frac{1}{3}}-h\left(\frac{E_{i}-E_{c}}{3 R^{\prime}}-300\right)\right] e^{-\frac{f E_{i}}{E_{m}-E_{i}}}
$$

where $p$ is the pressure, $V$ is the relative vulume, $E c$ is the cold compression energy: 
$E_{c}(x)=\int_{0}^{x} p d x-\frac{900 R^{\prime} \exp (a x)}{(1-x)^{2\left(\gamma_{0}-a-1 / 2\right)}}, x=1-V$

and $E m$ is the melting energy:

$$
E_{m}(x)=E_{c}(x)+3 R^{\prime} T_{m}(x)
$$

which is in terms of the melting temperature $\operatorname{Tm}(x)$ :

$T_{m}(x)=\frac{T_{m o} \exp (2 a x)}{V^{2\left(\gamma_{0}-a-1 / 3\right)}}$

In the above equation $R^{\prime}$ is defined by

$$
R^{\prime}=\frac{R \rho}{A}
$$

where $R$ is the gas constant and $A$ is the atomic weight.

The yield strength $\sigma_{y}$ is given by:

$\sigma_{y}=\sigma_{0}^{\prime}\left[1+b^{\prime} p V^{1 / 3}-h\left(\frac{E_{i}-E_{c}}{3 R^{\prime}}-300\right)\right] e^{-\frac{f E_{i}}{E_{m}-E_{i}}}$

if $E m$ exceeds $E i$. Here, $\sigma_{0}^{\prime}$ is given by:

$$
\sigma_{0}^{\prime}=\sigma_{0}\left[1+\beta\left(\gamma_{i}+\bar{\varepsilon}^{p}\right)\right]^{n}
$$

where $\sigma_{0}$ is the initial yield stress and $\gamma_{i}$ is the initial plastic strain.

Johnson Cook model expresses the flow stress as

$$
\sigma_{y}=\left(A+B \bar{\varepsilon}^{p^{n}}\right)\left(1+C \ln \&^{*}\right)\left(1-T^{*^{*}}\right)
$$

where

$$
\begin{aligned}
& A, B, C, n, \text { and } m=\text { input constants } \\
& \bar{\varepsilon}^{p} \text { effective plastic strain } \\
& T^{*} \text { homologous temperature }
\end{aligned}
$$

The High Explosive Burn model allows the modeling of the detonation of a high explosive. In addition an equation of state must be defined, such as JWL equation of state.

In the Plastic Kinematic model, strain rate is accounted for using the Cowper and Symonds model which scales the yield stress with the factor

$$
1+\left(\frac{\dot{\varepsilon}}{C}\right)^{1 / p}
$$

where $\dot{\varepsilon}$ is the strain rate. The yield stress is given by

$$
\sigma_{y}=\left[1+\left(\frac{\dot{\varepsilon}}{C}\right)^{1 / p}\right]\left(\sigma_{0}+\beta^{\prime} E_{p} \varepsilon_{e f f}^{p}\right)
$$

where $\quad \sigma_{0}$ is the initial yield stress;

$C$ and $P$ are Cowper-Symons strain rate parameter; $\varepsilon_{e f f}$ is the effective plastic strain; $\beta^{\prime}$ is the harden constant;

$E_{p}$ is the plastic harden modulus.

\subsection{Verification of the simulation model}

In the present study, we developed a model which is reasonable simplification, but a lot of complicated grid structures and the control parameters are involved. In order to verify the reliability of model analysis, we carried out relevant static scaled-down experiments.

In the experiment, the size ratio between the simulation model and the actual warhead is 1:1.25. Conditions of simulation are the same as that of real experiment. The results of simulation and experiment are shown in figure 4. The size of the hole is $400 \mathrm{~mm}$ in the experiment and $390 \mathrm{~mm}$ in the simulation, respectively. Relative error is $2.25 \%$. Therefore the analysis based on the simulation model is reliable.

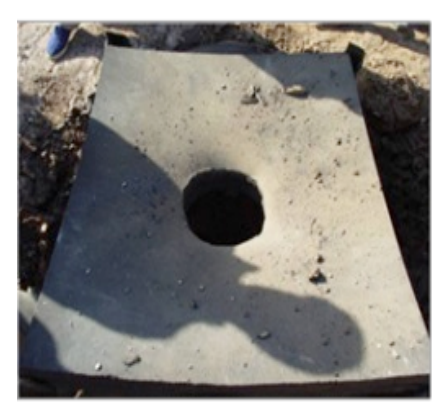

Experiment

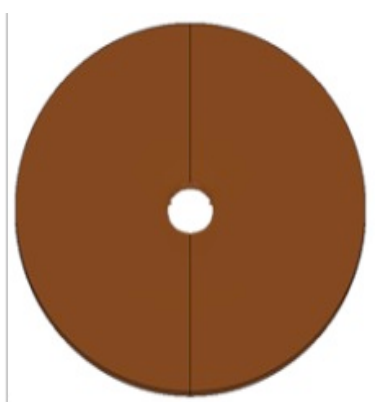

Simulation
Fig.4. Results of experiment and simulation of EFP impacting top deck

\subsection{Calculation cases}

Considering the three influence factors and technical requirement in application, we lists 15 calculation cases in table 2. Meanwhile, the "+" (or "-") symbol means the direction ("left" or "right") of the yaw angle under warhead with attack angle as shown in figure 5.

Table 2. Cases of simulation

\begin{tabular}{llll|}
\hline Case No. & $\begin{array}{c}\text { Attack velocity } \\
(\mathrm{m} / \mathrm{s})\end{array}$ & $\begin{array}{c}\text { Yaw angle } \\
\left({ }^{\circ}\right)\end{array}$ & $\begin{array}{c}\text { Attack angle } \\
\left({ }^{\circ}\right)\end{array}$ \\
\hline 1 & 750 & 0 & 0 \\
2 & 750 & 0 & 20 \\
3 & 750 & 3 & 0 \\
4 & 750 & +3 & 20 \\
5 & 750 & -3 & 20 \\
6 & 600 & 0 & 0 \\
7 & 600 & 0 & 20 \\
8 & 600 & 3 & 0 \\
9 & 600 & +3 & 0 \\
10 & 600 & -3 & 20 \\
11 & 500 & 0 & 0 \\
12 & 500 & 0 & 20 \\
13 & 500 & 3 & 0 \\
14 & 500 & +3 & 20 \\
15 & 500 & -3 & 20 \\
\hline
\end{tabular}




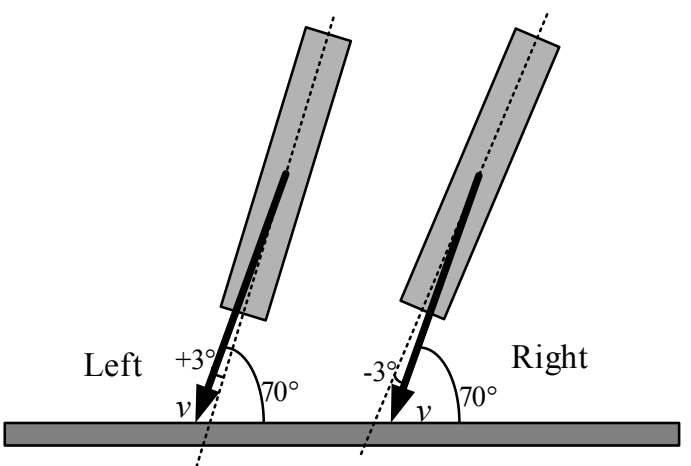

Fig.5. Difference between positive and negative yaw angle

\section{Results and discussion}

\subsection{The influence of precursor device detonation wave on} secondary projectile

The distance between the precursor shaped device and secondary projectile, $\mathrm{S}$, is a very important matching parameter in design. In general, $\mathrm{S}$ should be as small as possible, to ensure the secondary projectile pass through the hole on the top deck. Therefore, at first, this paper studied the influence of the precursor device detonation on the secondary projectile, and thereby determined the value of S. According to different values of $\mathrm{S}$, simulation calculations were carried out. The maximum stress and the velocity change of secondary projectile were used to evaluate its safety and flight stability, and then, the $\mathrm{S}$ was determined.

We analyzed the $\mathrm{S}$ effects with different values. As shown in figure 6 , when $S=30 \mathrm{~mm}$, the maximum stress of secondary projectile body is $1.41 \mathrm{GPa}$. This stress is near the strength of the projectile material and potentially will lead to projectile body damage; at the same time, its velocity change is $60.3 \mathrm{~m} / \mathrm{s}$, which indicates that the influence on the secondary projectile is evident. When $\mathrm{S}=35 \mathrm{~mm}$, the maximum stress of secondary projectile body is $1.05 \mathrm{GPa}$, and its velocity change is $40.6 \mathrm{~m} / \mathrm{s}$. Under this condition, the secondary projectile is safe and flies stably. Therefore, with comprehensive assessment, $\mathrm{S}$ is set to $35 \mathrm{~mm}$.

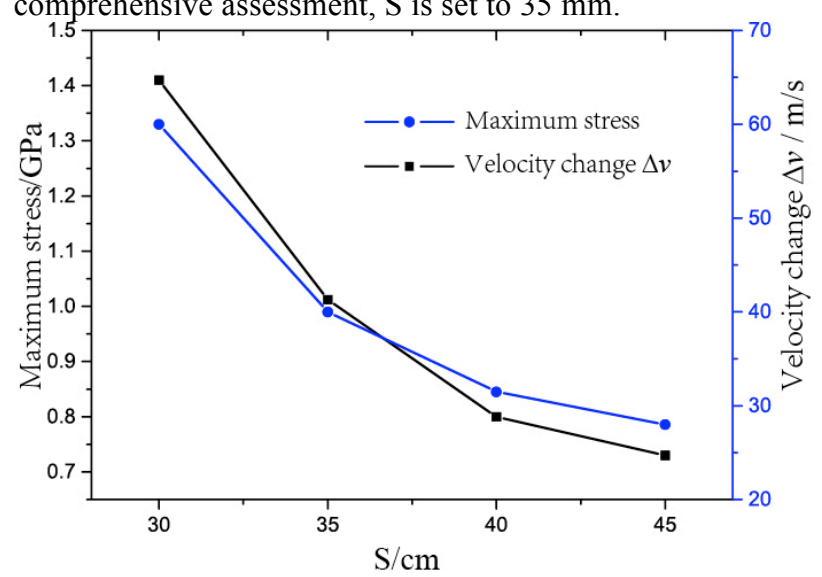

Fig.6. The maximum stress and velocity loss of the following warhead according to $\mathrm{S}$

\subsection{The influence of flying posture on EFP penetration}

The EFP formed by precursor shaped device is the most important penetrating power of the whole warhead, which affects secondary projectile following across the top deck and destroys the targets directly. Because the structure of precursor device is finished, the EFP shape is changeless.
The results indicate that the EFP shape is stable $200 \mu$ s after the detonation, and its velocity changes slightly. The EFP shape is shown in figure 7 , and the diameter of the main body is $496 \mathrm{~mm}$. Its velocity is $2407 \mathrm{~m} / \mathrm{s} 、 2493 \mathrm{~m} / \mathrm{s}$ 、 $2639 \mathrm{~m} / \mathrm{s}$, when the attack velocity is $500 \mathrm{~m} / \mathrm{s} 、 600 \mathrm{~m} / \mathrm{s}$ 、 $750 \mathrm{~m} / \mathrm{s}$, respectively.

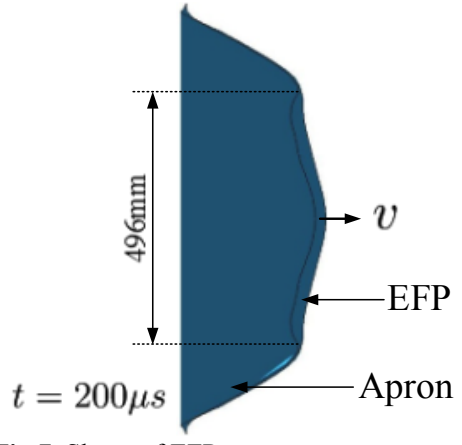

Fig.7. Shape of EFP

The EFP penetrates the top deck to form a hole for the secondary projectile, as shown in figure 8 (Case 1). Because of its high velocity during penetration, several big fragments were used to ensure the secondary projectile safe.

The hole on the top deck is oval when the tandem warhead with attack angle, and its minor axis is the diameter of EFP. However, projection on the normal plane of axis needs to be considered, namely, the equivalent diameter which is less than the long axis (Figure 9). The equivalent diameter $\phi_{E}$ equals $\phi_{0} \sin 70^{\circ}$, where $\phi_{0}$ is the long axis of the oval hole.
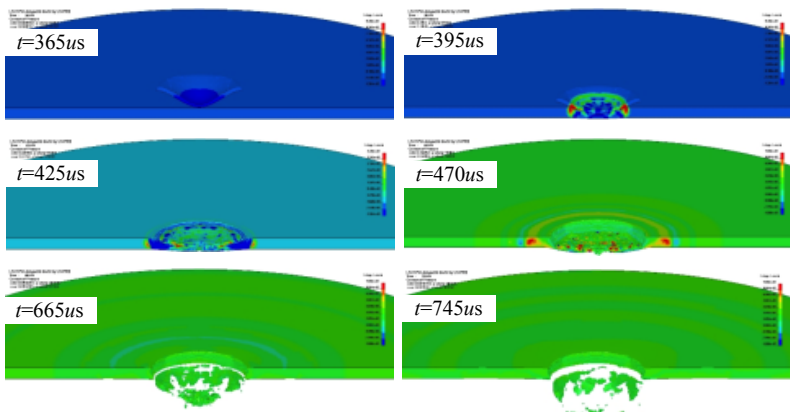

Fig.8. Vertical penetration of EFP against top deck

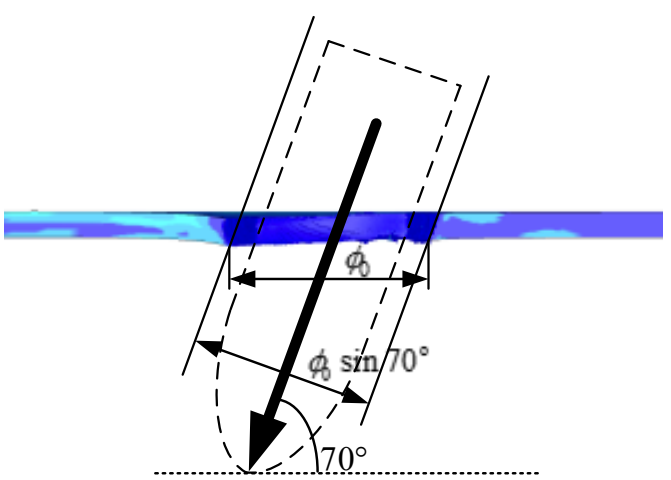

Fig.9. Equivalent diameter of hole

\subsubsection{The influence of attack velocity on equivalent diameter}

Change of attack velocity mainly affects the EFP velocity, but not EFP shape. The diameter of the hole on the top deck only is determined by EFP velocity. Calculation results of all cases are shown in table 3 . Without attack angle and yaw 
angle, the equivalent diameter is $496 \mathrm{~mm}, 500 \mathrm{~mm}, 508 \mathrm{~mm}$, when the attack velocity is $500 \mathrm{~m} / \mathrm{s}, 600 \mathrm{~m} / \mathrm{s}, 750 \mathrm{~m} / \mathrm{s}$, respectively, and the variation is less than $1.06 \%$. Under conditions with the same attack angle and yaw angle, similar phenomenon is shown in figure 7 . It can be seen that the EFP could maintain its shape well and have adequate kinetic energy to penetrate the top deck. The equivalent diameter is not sensitive to attack velocity, and the warhead adapts well with detonation height (distance between detonation point of precursor shaped device and the target).

\subsubsection{The influence of attack angle on equivalent diameter}

The attack angle is the angle between the velocity vector of the warhead and the normal vector of the top deck surface. When the attack angle equals $20^{\circ}$, the penetration process would be much hard, because the stress is asymmetrical and the equivalent thickness of top deck becomes bigger. As shown in table 3 and figure 10, the difference between with attack angle and without attack angle is equivalent. With the same attack velocity, the equivalent diameter under conditions without attack angle is obviously bigger than without that.

\subsubsection{The influence of yaw angle on equivalent diameter}

The influence rule of yaw angle on EFP penetrating performance is shown in figure 10. The equivalent diameter decreased when yaw angle does not equal zero and attack angle equals zero. If the attack angle is not zero, equivalent diameter under the condition with "left" yaw angle is bigger than that without yaw angle, which is on the contrary to that with "right" yaw angle. On the whole, if the absolute value of yaw angle is not more than $3^{\circ}$, the EFP penetrating performance would be guaranteed. The results indicate that the equivalent diameter range is less than $2 \%$. Even so, it is better to restrict the yaw angle.

Table 3. Equivalent diameter of holes under different cases

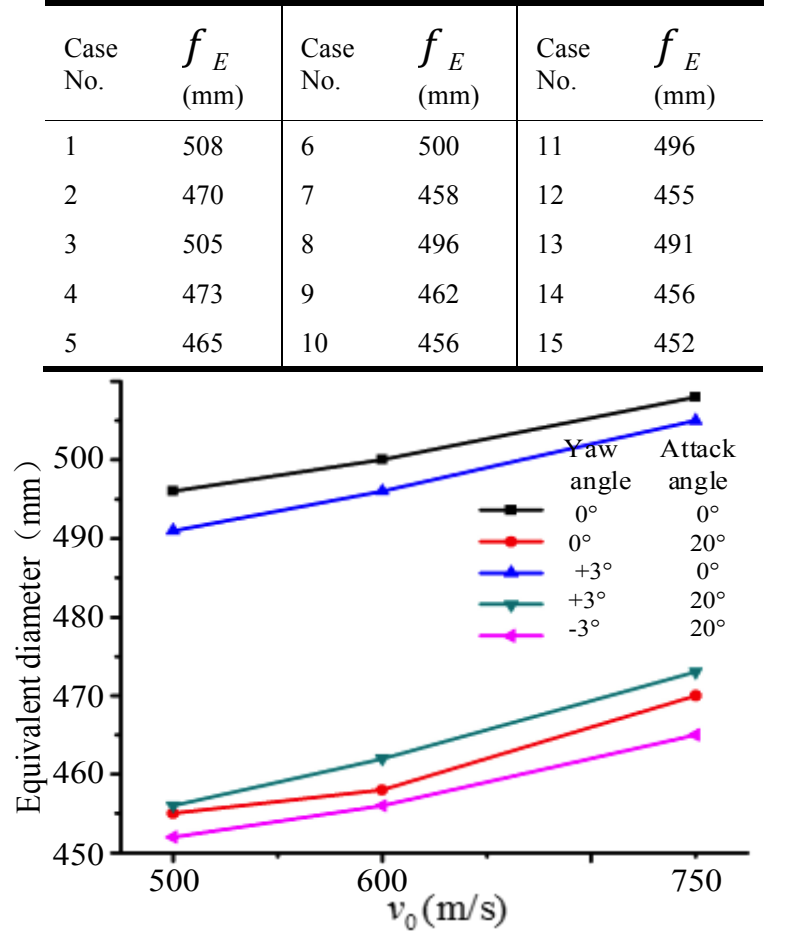

Fig.10. Equivalent diameter of hole under different warhead velocities

\subsection{The influence of flying posture on secondary} projectile following

In the 15 calculation cases, the equivalent diameter varies between $452 \mathrm{~mm}$ and $508 \mathrm{~mm}$ (Table 3), which is bigger than the diameter of secondary projectile. However, whether the secondary projectile would fly across the hole successfully, its own flying posture is much more important. When attack angle equals zero, secondary projectile would fly across the hole without touching the top deck, as shown in figure 11(a), and the existence of the yaw angle could be ignored. When attack angle equals $20^{\circ}$, secondary projectile would fly across the hole with touching the top deck. In this case, due to the effects of forces, secondary projectile may change its flying posture to be worse. To remit this situation, on the one hand, one possibility is that the equivalent diameter should be big enough; on the other hand, the strength of secondary projectile should be high enough. Only in this case, the second projectile could fly stable and would not be damaged. As shown in figure 11(b), under the condition with yaw angle, secondary projectile would impact the top deck. When secondary projectile impact the top deck while flying across the hole (Figure 12, Case 4), 6 elements were selected to study the stress of secondary projectile body during following process. The yield limit of secondary projectile body is $800 \mathrm{MPa}$, and the strength stress is up to $1.5 \mathrm{GPa}$. The 3147398 element has the maximum stress which is near to $800 \mathrm{MPa}$. Thus it can be seen that the secondary projectile would fly across the hole without any damage and its flying posture would also maintained well.

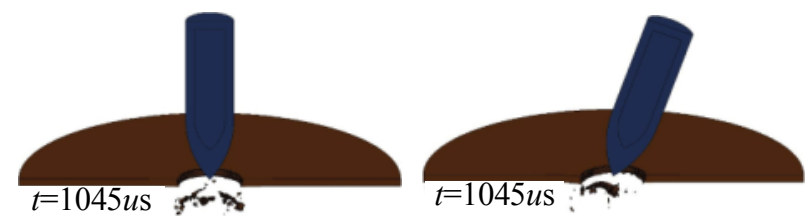

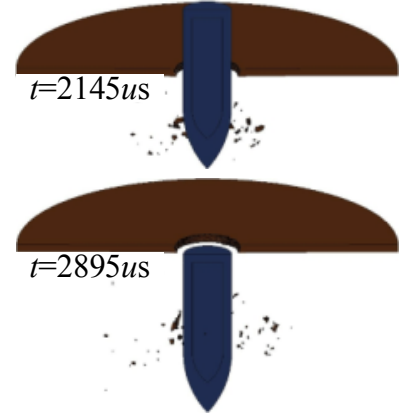

(a) Case 1

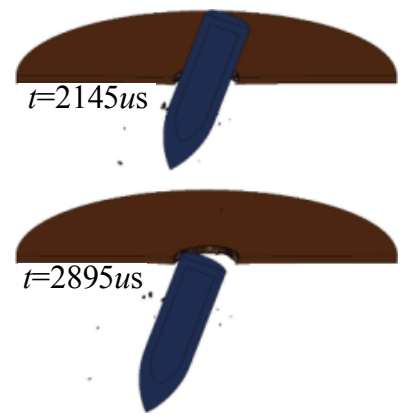

(b) Case 2
Fig.11. Following process of secondary projectile
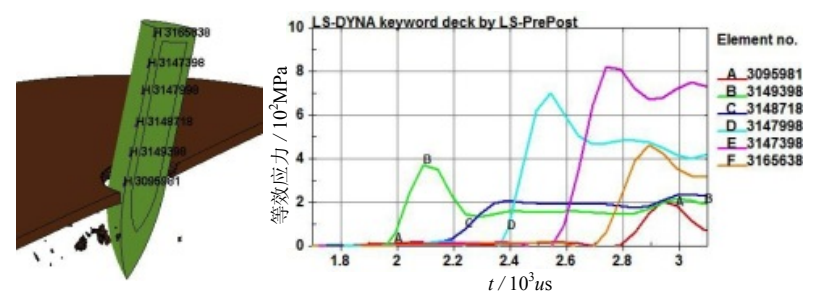

Fig.12. Stress of secondary projectile body during following process

\section{Conclusion}

In this study, we used a model established by LS-DYNA and carried out a series of simulation calculations for the complete assessment of the tandem warhead performance 
against military vessels. We showed that attack velocity has tiny influence on the equivalent diameter, and the equivalent diameter decreases as the attack angle increases; additionally, for the yaw changing from $-3^{\circ}$ to $+3^{\circ}$, on the one hand, the equivalent diameter varies slightly, on the other hand, because of its randomness, the yaw angle should be restricted. Furthermore, the secondary projectile was mainly affected by attack angle, slightly by the yaw angle. This novel tandem warhead has powerful penetrating performance and could ensure the secondary projectile fly across the top deck in order to achieve damage intentions.

\section{References}

1. Zhang T., Yang S., Xu S, “Technology Character and Development Tendency of Tandem Warhead", Industry Applications, Flight Missiles, 10(3), 2006, pp.487-494.

2. Wang S. H, Wang S. "The Research on Efficiency Assessment Method of Tandem Penetrating Blast Warhead". Journal of Projectiles, Rocket s, Missiles and Guidance, 30(1), 2010, pp.121124.

3. Men J., Jiang J. W, Yang J.. "Numerical Simulation and Experiments of Series EFP Forming and Penetration". Transactions of Beijing Institute of Technology, 30(2), 2010, pp.383-386.

4. Cui Z. G., Liu Z. C., Jing X. Y. "The Study of Forward Shaped Charge Structure for Tandem Warhead Based on LS-DYNA". Journal of Projectiles, Rocket s, Missiles and Guidance, 31(3), 2011, pp.113-115.

5. Wang L. X., Yuan B. H., Sun J. "Design and Experiment Study on the Precursory Shaped Charge of Tandem Warhead". Chinese Journal of Explosives \& Propellants, 34(5), 2011, pp.52-56.
6. Li W. B., Wang X. M., Li W. B.. "Feasibility research on the formation of a multimode explosively formed penetrator with single-point initiation". Explosion and Shock Waves, 31(2), 2011, pp.204-209.

7. Zheng Y., Wang X. M., Li W. B. "Effects of Liner Curvature Radius on Formation of Double-Layered Spherical Segment Charge Liner into Tandem Explosively Formed Projectile". Chinese Journal of High Pressure Physics, 23(4), 2009, pp.229-235.

8. S. V. Anfilets1, V. N. Shuts., "Optimizing the management of traffic light object based on natural algorithms", Journal Computer Modelling and New Technologies, 17(1), 2013, pp.7-13.

9. Liu Y., Ding Y. L. "The Modeling of Genetic and Tabu Search Algorithm Based BP Neural Network in the Risk Analysis of Investment", Journal of Digital Information Management, 11(1), 2013, pp. 391-399. 